\title{
SYNTHESIS AND PROPERTIES OF N-[2-(BENZOYLAMINO) (2-OXOINDOLIN-3-YLIDENE)ACETYL]AMINO ACIDS ETHYL ESTERS
}

\author{
O.O.Altukhov \\ National University of Pharmacy
}

Key words: synthesis; derivatives of 2-(benzoylamino)(1-R-2-oxo-1,2-dihydro-3H-indol-3-ylidene) acetic acids; azlactone; amino acids; ethyl esters

\begin{abstract}
Analysis of scientific and patent literature testifies that search of biologically active compounds among derivatives of 2-oxoindoline is prospective. They include well-known amino acids (tryptophane), neurohormone serotonine, a number of natural alkaloids and synthetic drugs (indomethacin, dimecarbin). For many years at the Analytical chemistry department of the National University of Pharmacy the extensive studies have been conducted in the field of development of synthetic methods and the study of physicochemical and biological characteristics of hetarylcarboxylic acids, in particular, 2-oxaindolineacetic acids and products of their transformation in order to search active and harmless medicines. The pharmacological research of (2-oxoindolin-3-ylidene)-2-oxoacetic acid derivatives allow making a conclusion that the highest nootropic activity is shown by substances containing amino acid esters moieties. Extending directions of the target-based search for biologically active compounds and basing on the previous studies it has been decided to include the fragments of amino acids into the core structure - 2-(benzoylamino)(2-oxoindolin-3-ylidene)-2-oxoacetic acid and to carry out the synthesis of $\mathrm{N}$-[2-(benzoylamino)(1-R-2-oxoindolin-3-ylidene)acetyl]amino acids ethyl esters. The structure of the compounds synthesized has been confirmed by the data of elemental analysis, IR- and ${ }^{1} H N M R-$ spectroscopy. In ${ }^{1} \mathrm{H}$ NMR spectrum of $\mathrm{N}$-[2-(benzoylamino)(1-R-2-oxoindolin-3-ylidene)acetyl]glycine the signal of the methylene group of the amino acid residue is observed as a duplet at $\delta 4.0 \mathrm{ppm}$. The signal of the carboxyl group proton in the spectrum of the acid is not observed as a result of deuterium exchange. The signal of $\mathrm{CH}_{2}$-group is shifted downfield $(4.08 \mathrm{ppm})$ for the ester. The signals of the ethoxy group are recognized as a quartet at $4.20 \mathrm{ppm}\left(2 \mathrm{H}, \mathrm{CH}_{2}\right)$ and a triplet at $1.21 \mathrm{ppm}\left(3 \mathrm{H}, \mathrm{CH}_{3}\right)$.
\end{abstract}

In Ukraine, as well as in the most countries all over the world, a cerebral stroke is one of the most frequent causes of disability and death rate. According to the research data there are 600 patients with the consequences of stroke per each 100 thousand of the population; $60 \%$ of them remain invalids. At the same time an active rehabilitation allows to decrease the degree of disability for patients that overcome the cerebral stroke and to turn them back to work.

Due to the fact that the native nootropic drugs nomenclature is significantly less than the nomenclature of this group of drugs available at foreign markets, and which is very often insufficient for necessities of medical practice satisfaction, the search of new nootropic drugs is an urgent task. Taking into consideration the reference sources the interest for chemistry of 2-oxoindolinacetic acids is conditioned by a significant biological activity of their derivatives [12, 14-16].

Lately the attempts of modification of different compounds by amino acids, which have a wide spectrum of the pharmacological activity and provide harmless and easily digestible form to other substances, are performed. In many cases they have the potentiating effect [17-20]. In addition, amino acids participate in the processes of nervous, vascular and other types of regulation of the body's functions.
The aim of our research is the synthesis of a new group of chemical compounds - N-[2-(benzoylamino) (1-R-2-oxoindolin-3-ylidene)acetyl]amino acids and their ethyl esters as potential biologically active substances [8-10].

An attempt to obtain N-[2-(benzoylamino)(1-R-2oxoindolin-3-ylidene)acetyl]amino acids using the traditional method leads to target products (2-4) with 45$50 \%$ yields. We offered the method with the use of methanol solution of potassium hydroxide and 1,4-dioxane, it allowed to increase the acid (2-4) yields up to $95 \%$ preserving a sufficient degree of their purity (Scheme 1).

The amino acids (2-4) synthesized are yellow crystalline substances with precious melting points after crystallization from 1,4-dioxane (Tab. 1); soluble when heating in ethanol, dioxane, DMFA, DMSO.

The pharmacological research of (2-oxoindolin-3ylidene)-2-oxoacetic acid derivatives allow making a conclusion that the highest nootropic activity is shown by substances containing amino acid esters moieties [5-7]. Taking this fact into account it was expedient to carry out the synthesis of $\mathrm{N}$-[2-(benzoylamino)(1-R2-oxoindolin-3-ylidene)acetyl]amino acid and, first of all, N-[2-(benzoylamino)(1-R-2-oxoindolin-3-ylidene)acetyl]glycine ethyl ester.

Compound (5) was obtained by esterification of N[2-(benzoylamino)(1-R-2-oxoindolin-3-ylidene)acetyl] 


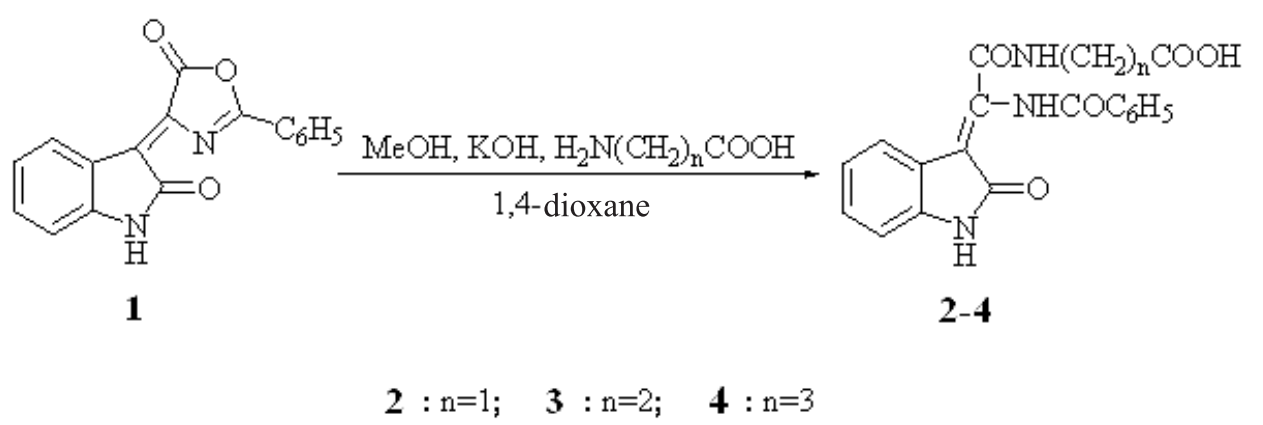

Scheme 1. Interaction of azlactone of 2-(benzoylamino)(2-oxoindolin-3-ylidene)acetic acid (1) with amino acids.

glycine (2) in absolute ethanol [3]. Ethyl esters of N[2-(benzoylamino)(1-R-2-oxoindolin-3-ylidene)acetyl] glycine (5.1-5.5) were synthesized by alkylation of the acid (2) in the presence of potassium carbonate in DMSO solution. These compounds can be also obtained by heating azlactones containing aliphatic substituents in 1 position of the oxoindoline cycle (1.1-1.5) with glycine ethyl ester hydrochloride in the ethanol media (Scheme 2).

$\mathrm{N}$-[2-(benzoylamino)(1-R-2-oxoindolin-3-ylidene) acetyl]glycine ethyl esters (5-5.5) after crystallization from 1,4-dioxane are yellow crystalline substances with precious melting points (Table 1), soluble in ethanol, dioxane, DMFA, DMSO.

The structure of compounds (5-5.5) were confirmed by the data of elemental analysis, IR- and ${ }^{1} \mathrm{H}$ NMRspectroscopies (Tab. 2).

IR-spectra of the compounds synthesized, except the stretching bands $v$ N-H 3332 and $3291 \mathrm{~cm}^{-1}$ (2-4) and $3297 \mathrm{~cm}^{-1}(\mathbf{5 - 5 . 5})$, have an increased intensity of stretching bands $\vee \mathrm{C}-\mathrm{H}$ in the range of $3150-2928 \mathrm{~cm}^{-1}$, and it indicates the presence of aliphatic substituents. The stretching band $v \mathrm{C}=\mathrm{O}$ at $1733(\mathbf{2 - 4})$ and $1737 \mathrm{~cm}^{-1}$ (5-5.5) testifies the presence of the carbonyl group in the structure bonded to the alkyl radical $[1,4]$.

In ${ }^{1} \mathrm{H}$ NMR spectrum of $\mathrm{N}$-[2-(benzoylamino)(1-R2-oxoindolin-3-ylidene)acetyl]glycine (2) the signal of the methylene group of amino acid is recognized as a duplet at $\delta 4.0 \mathrm{ppm}$. The signal of the carboxyl group proton in the spectrum of the acid is not observed as a result of the deuterium exchange. The signal of the $\mathrm{CH}_{2}$-group is shifted downfield (4.08 ppm) for the ester (5). The signals of the ethoxy-group are observed as a quartet at $4.20 \mathrm{ppm}\left(2 \mathrm{H}, \mathrm{CH}_{2}\right)$ and a triplet at $1.21 \mathrm{ppm}$ $\left(3 \mathrm{H}, \mathrm{CH}_{3}\right)[13]$.

\section{Experimental Part}

When studying the objects under research in order to confirm the structure and purity of the substances synthesized the physical and chemical methods described in the State Pharmacopeia of Ukraine were used [2].

Melting points were determined by the capillary method on a "PTM (M)" apparatus. The elemental analysis of Nitrogen was carried out on an automatic analyser "CNH", model EA 1108 "Carlo Erba".

IR-spectra were registered on a "Tensor 27" apparatus in $\mathrm{KBr}$ tablets, the concentration of the substance $-1 \%$.

${ }^{1} \mathrm{H}$ NMR spectra of the compounds synthesized were recorded on a Varian Mercury VX-200 spectrophotometer $(200 \mathrm{MHz})$. The solvent was DMSO-D 6 , the internal standard was tetramethylsilane (TMS). Chemical shifts are given at the ppm scale.

The data of elemental analysis correspond to the calculated one.

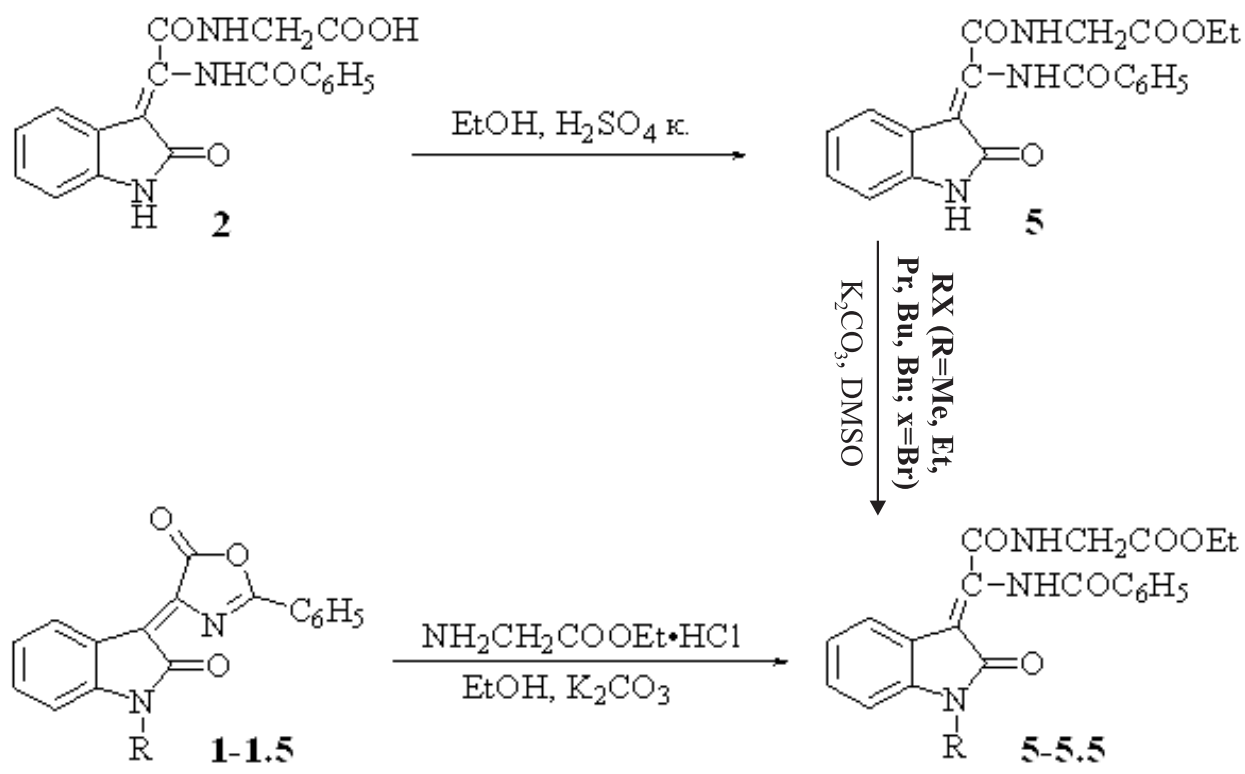

Scheme 2. Synthesis of N-[2-(benzoylamino)7(1-R-2-oxoindolin-3-ylidene)acetyl]glycine ethyl ester (5-5.5). 
Characteristics of N-[2-(benzoylamino)(1-R-2-oxoindolin-3-ylidene)acetyl]amino acids (2-4) and ethyl esters (5-5.5)

\begin{tabular}{|c|c|c|c|c|}
\hline Compound & Molecular formula & $\frac{\text { Found } \mathrm{N}, \%}{\text { Calculated } \mathrm{N}, \%}$ & Melting point, ${ }^{\circ} \mathrm{C}$ & Yield, \% \\
\hline 2 & $\mathrm{C}_{19} \mathrm{H}_{15} \mathrm{~N}_{3} \mathrm{O}_{5}$ & $\frac{11.51}{11.50}$ & $250-252$ & 95 \\
\hline 3 & $\mathrm{C}_{20} \mathrm{H}_{17} \mathrm{~N}_{3} \mathrm{O}_{5}$ & $\frac{11.07}{11.08}$ & $250-252$ & 93 \\
\hline 4 & $\mathrm{C}_{21} \mathrm{H}_{19} \mathrm{~N}_{3} \mathrm{O}_{5}$ & $\frac{10.66}{10.68}$ & $222-223$ & 92 \\
\hline 5 & $\mathrm{C}_{21} \mathrm{H}_{19} \mathrm{~N}_{3} \mathrm{O}_{5}$ & $\frac{10.65}{10.68}$ & $248-250$ & 92 \\
\hline 5.1 & $\mathrm{C}_{22} \mathrm{H}_{21} \mathrm{~N}_{3} \mathrm{O}_{5}$ & $\frac{10.34}{10.31}$ & $232-234$ & 84 \\
\hline 5.2 & $\mathrm{C}_{23} \mathrm{H}_{23} \mathrm{~N}_{3} \mathrm{O}_{5}$ & $\frac{9.98}{9.97}$ & $174-176$ & 81 \\
\hline 5.3 & $\mathrm{C}_{24} \mathrm{H}_{25} \mathrm{~N}_{3} \mathrm{O}_{5}$ & $\frac{9.63}{9.65}$ & $160-162$ & 88 \\
\hline 5.4 & $\mathrm{C}_{25} \mathrm{H}_{27} \mathrm{~N}_{3} \mathrm{O}_{5}$ & $\begin{array}{l}9.37 \\
.35 \\
\end{array}$ & $153-155$ & 86 \\
\hline 5.5 & $\mathrm{C}_{28} \mathrm{H}_{25} \mathrm{~N}_{3} \mathrm{O}_{5}$ & $\frac{8.67}{8.69}$ & 196-198 & 99 \\
\hline
\end{tabular}

N-[2-(benzoylamino)(1-R-2-oxoindolin-3-ylidene)acetyl]glycine (2)<smiles>O=C(CNC(=O)c1ccccc1)NC1C(=O)Nc2ccccc21</smiles>

To the solution of $0.3 \mathrm{~g}(0.005 \mathrm{~mol})$ of $\mathrm{KOH}$ in $10 \mathrm{~cm}^{3}$ of methanol add $0.4 \mathrm{~g}(0.005 \mathrm{~mol})$ of glycine. Transfer the mixture obtained into solution of $1.45 \mathrm{~g}$ $(0.005 \mathrm{~mol})$ of 3-(5-oxo-2-phenyl-1,3-oxazole-4(5H)ylidene)-1,3-dihydro-2H-indol-2-one) (1) in $10 \mathrm{~cm}^{3}$ of 1,4-dioxane. Heat the reaction mixture with a reflux on a water bath for $60 \mathrm{~min}$, then cool and transfer into water acidified by chloric acid. Filter the precipitate, wash with water, dry and crystallize from 1,4-dioxane. The

Table 2

${ }^{1} \mathrm{H}$ NMR spectra of N-[2-(benzoylamino)(1-R-2-oxoindolin-3-ylidene)acetyl]amino acids (2-4) and ethyl esters (5-5.5)

\begin{tabular}{|c|c|c|c|c|c|}
\hline \multirow[b]{2}{*}{ Compound } & \multicolumn{5}{|c|}{ Chemical shifts, $\delta$, ppm. } \\
\hline & $\begin{array}{l}\mathrm{NHCO} \\
(1 \mathrm{H}, \mathrm{c})\end{array}$ & $\begin{array}{c}\mathrm{NH} \text {-indol } \\
(1 \mathrm{H}, \mathrm{c})\end{array}$ & $\begin{array}{l}\mathrm{CONH} \\
(1 \mathrm{H}, \mathrm{T})\end{array}$ & Ar-H & Protons signals of other functional groups \\
\hline 2 & 12.89 & 11.05 & 9.18 & $8.01-6.75, \mathrm{~m}, 9 \mathrm{H}$ & $3.91\left(2 \mathrm{H}, \mathrm{d}, \mathrm{CH}_{2}\right)$ \\
\hline 3 & 12.81 & 11.01 & 8.91 & $8.02-6.81, m, 9 \mathrm{H}$ & $3.51\left(2 \mathrm{H}, \mathrm{m}, \mathrm{CH}_{2} \mathrm{CH}_{2}\right) ; 2.61\left(2 \mathrm{H}, \mathrm{t}, \mathrm{CH}_{2} \mathrm{CH}_{2}\right)$ \\
\hline 4 & 12.85 & 11.07 & 8.09 & $7.95-6.90, \mathrm{~m}, 9 \mathrm{H}$ & $\begin{array}{l}3.35\left(2 \mathrm{H}, \mathrm{m}, \mathrm{CH}_{2} \mathrm{CH}_{2} \mathrm{CH}_{2}\right) ; 2.27\left(2 \mathrm{H}, \mathrm{t}, \mathrm{CH}_{2} \mathrm{CH}_{2} \mathrm{CH}_{2}\right) ; \\
1.78\left(2 \mathrm{H}, \mathrm{m}, \mathrm{CH}_{2} \mathrm{CH}_{2} \mathrm{CH}_{2}\right)\end{array}$ \\
\hline 5 & 12.92 & 11.10 & 9.28 & $8.02-6.92, \mathrm{~m}, 9 \mathrm{H}$ & $4.09\left(4 \mathrm{H}, \mathrm{m}, \mathrm{NHCH}{ }_{2}+\underline{\mathrm{CH}}_{2} \mathrm{CH}_{3}\right) ; 1.21\left(3 \mathrm{H}, \mathrm{t}, \mathrm{CH}_{3}\right)$ \\
\hline 5.1 & 12.89 & & 9.21 & $8.02-6.82, \mathrm{~m}, 9 \mathrm{H}$ & $\begin{array}{l}4.11\left(4 \mathrm{H}, \mathrm{m}, \mathrm{NH}_{\mathrm{CH}}+\mathrm{OCH}_{2} \mathrm{CH}_{3}\right) ; 3.25\left(3 \mathrm{H}, \mathrm{s}, \mathrm{NCH}_{3}\right) ; \\
1.21\left(3 \mathrm{H}, \mathrm{t}, \mathrm{OCH}_{2} \underline{\underline{\mathrm{CH}}}_{3}\right)\end{array}$ \\
\hline 5.2 & 12.88 & - & 9.24 & $7.98-6.83, \mathrm{~m}, 9 \mathrm{H}$ & $\begin{array}{l}4.18\left(4 \mathrm{H}, \mathrm{m}, \mathrm{CH}_{2} \mathrm{COOCH}\right. \\
1.20\left(6 \mathrm{H}, \mathrm{m}, 2 \mathrm{CH}_{3}\right) ; 3.80\left(2 \mathrm{H}, \mathrm{t}, \mathrm{NCH}_{2}\right) ;\end{array}$ \\
\hline 5.3 & 12.89 & - & 9.23 & $7.95-6.81, \mathrm{~m}, 9 \mathrm{H}$ & $\begin{array}{l}4.12\left(4 \mathrm{H}, \mathrm{m}, \mathrm{CH}_{2} \mathrm{COOCH}_{2}\right) ; 3.80\left(2 \mathrm{H}, \mathrm{t}, \mathrm{NCH}_{2}\right) ; \\
1.65\left(2 \mathrm{H}, \mathrm{m}, \mathrm{CH}_{2} \mathrm{CH}_{2} \mathrm{CH}_{3}\right) ; 1.21\left(3 \mathrm{H}, \mathrm{t}, \mathrm{OCH}_{2} \mathrm{CH}_{3}\right) ; \\
0.89\left(3 \mathrm{H}, \mathrm{m}, \mathrm{CH}_{2} \mathrm{CH}_{2} \underline{\mathrm{CH}}_{3}\right)\end{array}$ \\
\hline 5.4 & 12.89 & - & 9.24 & $7.95-6.83, \mathrm{~m}, 9 \mathrm{H}$ & $\begin{array}{l}\left.\text { 4.20 (4H, m, } \underline{\mathrm{CH}}_{2} \mathrm{COOCH}_{2}\right) ; 3.75\left(2 \mathrm{H}, \mathrm{t}, \mathrm{NCH}_{2}\right) ; \\
1.67\left(2 \mathrm{H}, \mathrm{m}, \mathrm{NCH}_{2} \mathrm{CH}_{2}\right) ; 1.23\left(2 \mathrm{H}, \mathrm{m}, \mathrm{N}\left(\mathrm{CH}_{2}\right)_{2} \mathrm{CH}_{2}\right) ; \\
1.20\left(3 \mathrm{H}, \mathrm{t}, \mathrm{CCH}_{2} \underline{\mathrm{CH}}_{3}\right) ; 0.88\left(3 \mathrm{H}, \mathrm{t}, \mathrm{N}\left(\mathrm{CH}_{2}\right)_{3} \underline{\mathrm{CH}}_{3}\right)\end{array}$ \\
\hline 5.5 & 12.81 & - & 9.31 & $7.98-6.81, \mathrm{~m}, 14 \mathrm{H}$ & $\begin{array}{l}5.02\left(2 \mathrm{H}, \mathrm{s}, \mathrm{NCH}_{2}\right) ; 4.12\left(4 \mathrm{H}, \mathrm{m}, \underline{\mathrm{CH}}_{2} \mathrm{COOC \textrm {CH } _ { 2 }}\right) ; \\
1.19\left(3 \mathrm{H}, \mathrm{t}, \mathrm{CH}_{2} \mathrm{CH}_{3}\right)\end{array}$ \\
\hline
\end{tabular}


yield is $1.72 \mathrm{~g}(95 \%), \mathrm{m} . \mathrm{p} .250-252^{\circ} \mathrm{C}$. Compounds $(\mathbf{3 , 4})$ are obtained in the same manner.

$\mathrm{N}$-[2-(benzoylamino)(1-R-2-oxoindolin-3-ylidene)acetyl]glycine ethyl ester (5)

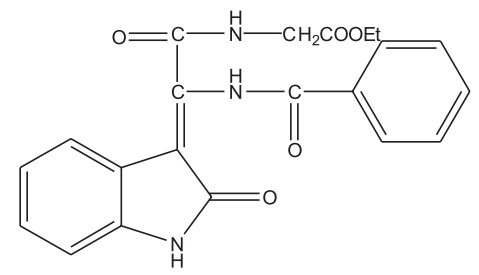

Method A. In a round-bottomed flask place $1.82 \mathrm{~g}$ (0.005 mol) of N-[2-(benzoylamino)(1-R-2-oxoindolin3-ylidene)acetyl]glycine (2), $15 \mathrm{~cm}^{3}$ of absolute ethanol and $0.2 \mathrm{ml}$ of the concentrated sulfuric acid. Heat the flask with a reflux and a drying tube on a water bath for $90 \mathrm{~min}$. In 12 hours filter the precipitate formed, wash with water, dry and crystallize from 1,4-dioxane. The yield is $1.8 \mathrm{~g}(92 \%)$, m.p. $248-250^{\circ} \mathrm{C}$. Compounds (5.15.5) are obtained in the same manner.

Method B. To $1.45 \mathrm{~g}(0.005 \mathrm{~mol})$ solution of 3-(5oxo-2-phenyl-1,3-oxazole-4(5H)-ylidene)-1,3-dihydro-
2H-indol-2-one) (1) in $15 \mathrm{~cm}^{3}$ of ethanol add $1.4 \mathrm{~cm}^{3}$ $(0.005 \mathrm{~mol})$ of triethylamine and $1.04 \mathrm{~g}(0.005 \mathrm{~mol})$ of glycine ethyl ester hydrochloride. Heat the reaction mixture with a reflux on a water bath for $60 \mathrm{~min}$, then cool and transfer into water acidified by chloric acid. Filter the precipitate, wash with water, dry and crystallize from 1,4-dioxane. The yield is $1.8 \mathrm{~g}(92 \%)$, m.p. $248-250^{\circ} \mathrm{C}$. The mixed sample of the compounds obtained by A and B methods does not possess melting point depression, their ${ }^{1} \mathrm{H}$ NMR-spectra are identical.

CONCLUSIONS

1. The method for obtaining of $\mathrm{N}$-[2-(benzoylamino) (1-R-2-oxoindolin-3-ylidene)acetyl]amino acids with the use of methanol solution of potassium hydroxide and 1,4-dioxane has been developed, and it allows to increase the yields of target products up to $95 \%$ preserving a sufficient degree of their purity. Ethyl esters of N-[2-(benzoylamino)(1-R-2-oxoindolin-3-ylidene)acetyl]glycine have been synthesized.

2. The structure of the compounds synthesized has been proven by the data of elemental analysis, IR- and ${ }^{1} \mathrm{H}$ NMR-spectroscopy.

\section{REFERENCES}

1. Беллами Л. Инфракрасные спектры сложных молекул. - М.: Изд-во иностранной литературы, 1963. $590 \mathrm{c}$.

2. Державна фармакопея Украйни / Державне підприємство «Науково-експертний фармакопейний центр». 1 -е вид. - Х.: ООО «РИРЕГ», 2001. - $556 c$.

3. Колісник С.В., Болотов В.В., Алтухов О.О., Шиикіна С.В. // ЖОФХ. - 2010. - Т. 8, вип. 3 (31). - С. 65-70.

4. Наканиси К. Инфракрасные спектры и строение органических соединений. - М.: Мир, 1965. - 216 с.

5. Пат. на корисну модель №38064 (2008) Україна // Б.В. - 2008. - №24.

6. Пат. на винахід №89542 (2010) Украӥна // Б.В. - 2010. - №3.

7. Пат. на винахід №90357 (2010) Украӥна // Б.В. - 2010. - №8.

8. Шатілов О.В., Штриголь С.Ю., Колісник С.В. та ін. // Актуальні проблеми сучасної медицини: Вісник Украйнської медичної стоматологічної академії. - 2009. - Т. 9, Вип. 2(26). - С. 139-142.

9. Штриголь С.Ю., Стіхарний О.О., Колісник С.В. та ін. // Вісник фармаиї̈. - 2008. - №4 (56). - С. 75-77.

10. Штриголь С.Ю., Стіхарний О.О., Колісник С.В. та ін. // Вісник фармаиії. - 2008. - №3 (55). - С. 60-63.

11. Andreani A., Rambaldi M., Locatelli A. et al. // Acta Pharm. Nord. - 1991. - Vol. 3, №1. - P. 5-8.

12. Bouchikhi F., Rossignil E., Sancelme M. et al. // Eur. J. Med. Chem. - 2008. - №43. - P. 2316-2322.

13. Breitmaier E. Structure elucidation by NMR in organic chemistry. $-3^{\text {rd }}$ ed. - Chichester: John Wiley \& Sons, 2002. $-258 p$.

14. Gruda J. // Can. J. Chem. - 1972. - Vol. 50, №1. - P. 18-23.

15. Hodjes R., Shannon J.S., Jamieson W.D., Taylor A. // Can. J. Chem. - 1968. - Vol. 46, №13. - P. 2189-2194.

16. Kenichi O., Ryota Sh., Takashi O. et al. // Bioorg. \& Med. Chem. - 2008. - Vol. 16, №23. - P. 10001-10012.

17. Nagarajan K., Talwaker P., Goud A. et al. // Ind. J. Chem. «B». - 1998. - Vol. 27, №12. - P. 1113-1123.

18. Stefanovich G., Mihailovich S. // Glasnik Khem. Drushiva. - 1959. - №22. - P. 459-471.

19. Terzioglu N., Karali N., Gürsoy A. et al. // ARKIVOC. - 2006. - Vol. 1. - P. 109-115.

20. Wikerson W., Kergaye A., Tam W. // J. Med. Chem. - 1993. - Vol. 36, №20. - P. 2899-2907.

СИНТЕЗ І ВЛАСТИВОСТІ ЕТИЛОВИХ ЕСТЕРІВ N-[2-(БЕНЗОЇЛАМІНО)(2-ОКСОІНДОЛІН3-ІЛІДЕН)АЦЕТИЛ]АМІНОКИСЛОТ

O.O.Алтухов

Ключові слова: синтез; похідні 2-(бензоїламіно)(1-R-2-оксо-1,2-дигідро-ЗН-індол-3-іліден) оцтових кислот; аз лактон; амінокислоти; етилові естери

Аналіз наукової та патентної літератури свідчить про перспективність пошуку біологічно активних сполук серед похідних 2-оксоіндоліну, серед яких відомі амінокислоти (триптофан), 
нейрогормон серотонін, ряд природних алкалоїдів і синтетичних лікарських засобів (індометацин, димекарбін). На кафедрі аналітичної хімії Національного фрармацевтичного університету протягом багатьох років проводяться широкі дослідження в галузі розробки методів синтезу та вивчення фозико-хімічних і біологічних властивостей гетерилкарбонових кислот, зокрема, 2-оксоіндоліноцтових кислот і продуктів їх перетворення з метою пошуку активних та нешкідливих лікарських засобів. Фармакологічні дослідження похідних (2-оксоіндолін-3-іліден)2-оксіоцтової кислоти дозволили зробити висновок, що найбільшу ноотропну активність проявляють речовини, до складу яких входять залишки естерів амінокислот. Розширюючи напрямки цілеспрямованого пошуку БАР, а також спираючись на попередні дослідження, ми вирішили до базової структури 2-(бензоїламіно)(2-оксоіндолін-3-іліден)оцтової кислоти ввести фррагменти амінокислот і здійснити синтез етилових естерів N-[2-(бензоїламіно)(1-R-2оксоіндолін-3-іліден)ацетил]амінокислот. Будову сполук доведено даними елементного аналізу, І4- та ЯМР ${ }^{1} \mathrm{H}$-спектроскопії. В ЯMP ${ }^{1} \mathrm{H}$-спектрі N-[2-(бензоїламіно)(1-R-2-оксоіндолін-3іліден)ацетил]гліцину сигнал метиленової групи амінокислотного залишку проявляється у вигляді дублету при б 4,0 м.ч. Сигнал протону карбоксильної групи у спектрі зазначеної кислоти не спостерігається внаслідок дейтерообміну. Для естеру сигнал $\mathrm{CH}_{2}$-групи зміщується у більш слабке поле 4,08 м.ч. Сигнали етоксильної групи мають вигляд квартету при 4,20 м.ч. $\left(2 \mathrm{H}, \mathrm{CH}_{2}\right)$ та триплету при 1,21 м.ч. $\left(3 \mathrm{H}, \mathrm{CH}_{3}\right)$.

\section{СИНТЕЗ И СВОЙСТВА ЭТИЛОВЫХ ЭФИРОВ N-[2-(БЕНЗОИЛАМИНО)(2-ОКСОИНДОЛИН- 3-ИЛИДЕН)АЦЕТИЛЈАМИНОКИСЛОТ \\ А.А.Алтухов}

Ключевые слова: синтез; производные 2-(бензоиламино)(1-R-2-оксо-1,2-дигидро-3Н-индол3-илиден)уксусных кислот; азлактон; аминокислоты; этиловые эфиры

Анализ научной и патентной литературы свидетельствует о перспективности поиска биологически активных соединений среди производных 2-оксоиндолина, среди которых известные аминокислоты (триптофран), нейрогормон серотонин, ряд природных алкалоидов и синтетических лекарственных средств (индометацин, димекарбин). На кафредре аналитической химии Национального фрармацевтического университета в течение многих лет проводятся широкие исследования в области разработки методов синтеза и изучение фризико-химических и биологических свойств гетерилкарбоновых кислот, в частности, 2-оксоиндолинуксусных кислот и продуктов их преобразования с целью поиска активных и безвредных лекарственных средств. Фармакологические исследования производных (2-оксоиндолин-3-илиден)-2-оксиуксусной кислоты позволили сделать вывод, что наибольшую ноотропную активность проявляют вещества, в состав которых входят остатки эфриров аминокислот. Расширяя аспекты целенаправленного поиска БАВ, а также опираясь на предыдущие исследования, мы решили в базовую структуру 2-(бензоиламино)(2-оксоиндолин-3-илиден)уксусной кислоты ввести фррагменты аминокислот и осуществить синтез этиловых эфриров $\mathrm{N}$-[2-(бензоиламино)(1-R-2-оксоиндолин-3-илиден)ацетил]аминокислот. Строение соединений доказано данными элементного анализа, ИК- и ЯМР ${ }^{1} \mathrm{H}$-спектроскопии. В ЯМР ${ }^{1} \mathrm{H}$ спектте $\mathrm{N}$-[2(бензоиламино)(1-R-2-оксоиндолин-3-илиден)ацетил]елицина сигнал метиленовой группы аминокислотного остатка проявляется в виде дублета при $\delta$ 4,0 м.ч. Сигнал протона карбоксильной группы в спектре указанной кислоты не наблюдается вследствие дейтерообмена. Для эфрира сигнал $\mathrm{CH}_{2}$-группы смещается в более слабое поле 4,08 м.ч. Сигналы этоксильной группы имеют видे квартета при 4,20 м.ч. (2H, $\left.\mathrm{CH}_{2}\right)$ и триплета при 1,21 м.ч. (3H, $\left.\mathrm{CH}_{3}\right)$. 